\title{
MISC, an instrument for multi-dimensional spectroscopy
}

\author{
F. Stolpe and F. Kneer \\ Universitäts-Sternwarte, Geismarlandstraße 11, D-37083 Göttingen, Germany \\ e-mail: fstolpe@uni-sw.gwdg.de
}

Received December 24, 1997; accepted February 16, 1998

\begin{abstract}
A new instrument for multi-dimensional spectroscopy at the Gregory Coudé Telescope (GCT) at the Observatorio del Teide/Tenerife is presented. The system, consisting of a scanning device, a Stokes $-V$ polarimeter and a suitable CCD system, performs fast spatial scanning. It preserves the good polarization properties of the telescope by using a Bowen compensator. We describe the instrument, the different modes of operation, and how it is controlled and give some performance data.
\end{abstract}

Key words: instruments: spectrographs; polarimeters Sun: general

\section{Introduction}

Many investigations of solar structures require multi-dimensional spectroscopy, so it is a subject of intense instrumental developments. Usually, multi-dimensionality implies the spectroscopy in two spatial coordinates. Additional information such as the state of polarization and the temporal evolution are often measured too.

Data obtained by such an instrument can be described as a cube with two spatial dimensions and one dimension of wavelength (Fig. 1). Investigations of the state of polarization add for each measurement taken such a cube. A Stokes $-V$ polarimeter for example splits the original cube into two sub-cubes. Time series are giving such a set of data cubes for each time a measurement is made. Hence, the amount of data obtained can thus become very large.

Classical spectroscopy yields single slices of this cube, for example: slit spectra, spectro-heliograms, narrow band filtergrams, etc... Several methods are known to obtain complete data cubes. Taking two-dimensional images while scanning through the wavelength is done with a Fabry-Perot interferometer (FPI, Bonaccini et al. 1989; Bendlin et al. 1992). An instrument with similar capabilities as a FPI is the Solar Optical Universal Polarimeter

Send offprint requests to : F. Stolpe

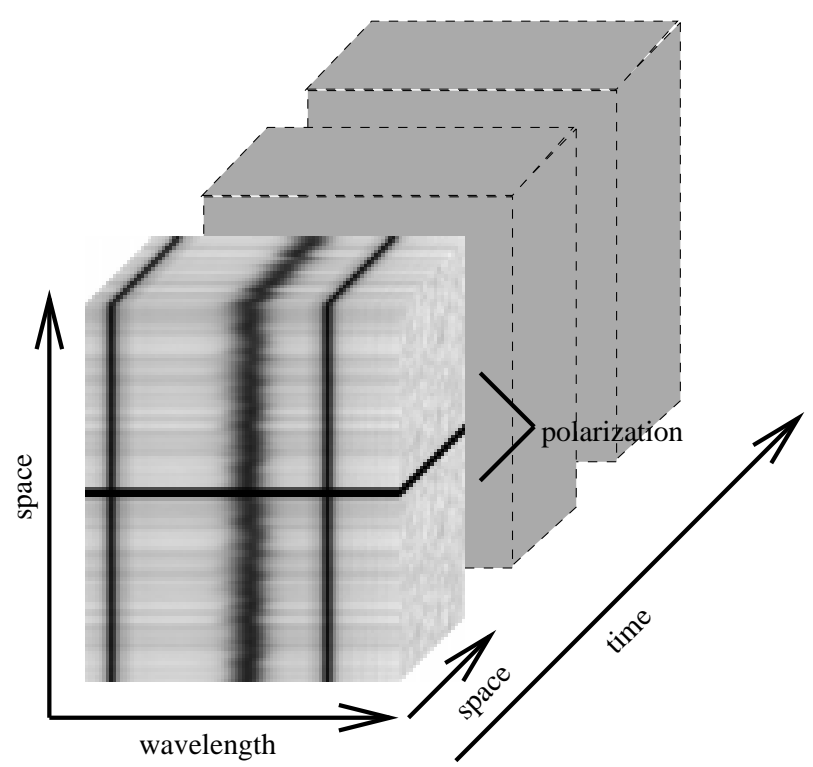

Fig. 1. The hyper cube structure of multidimensional spectra. The front cube was derived from observational data

(SOUP, Title 1984; Title et al. 1986), based on a fast tunable birefringent filter.

The Multi-channel Subtractive Double-Pass Spectrograph (MSDP, Mein 1977, 1991) allows to obtain simultaneous spatial and wavelength information. Scanning in one spatial direction and obtaining slit spectra, with the slit perpendicular to the scanning direction, is another method (e.g. Johannesson et al. 1987; Ballesteros et al. 1996).

All these methods have advantages and disadvantages. Some instruments are difficult to operate, others mix information from different coordinates which have to be disentangled during data reduction. If high spectral resolution is needed, spatial scanning will be the choice. In this paper we present such a device: the Micro-Image-Scanner (MISC). 

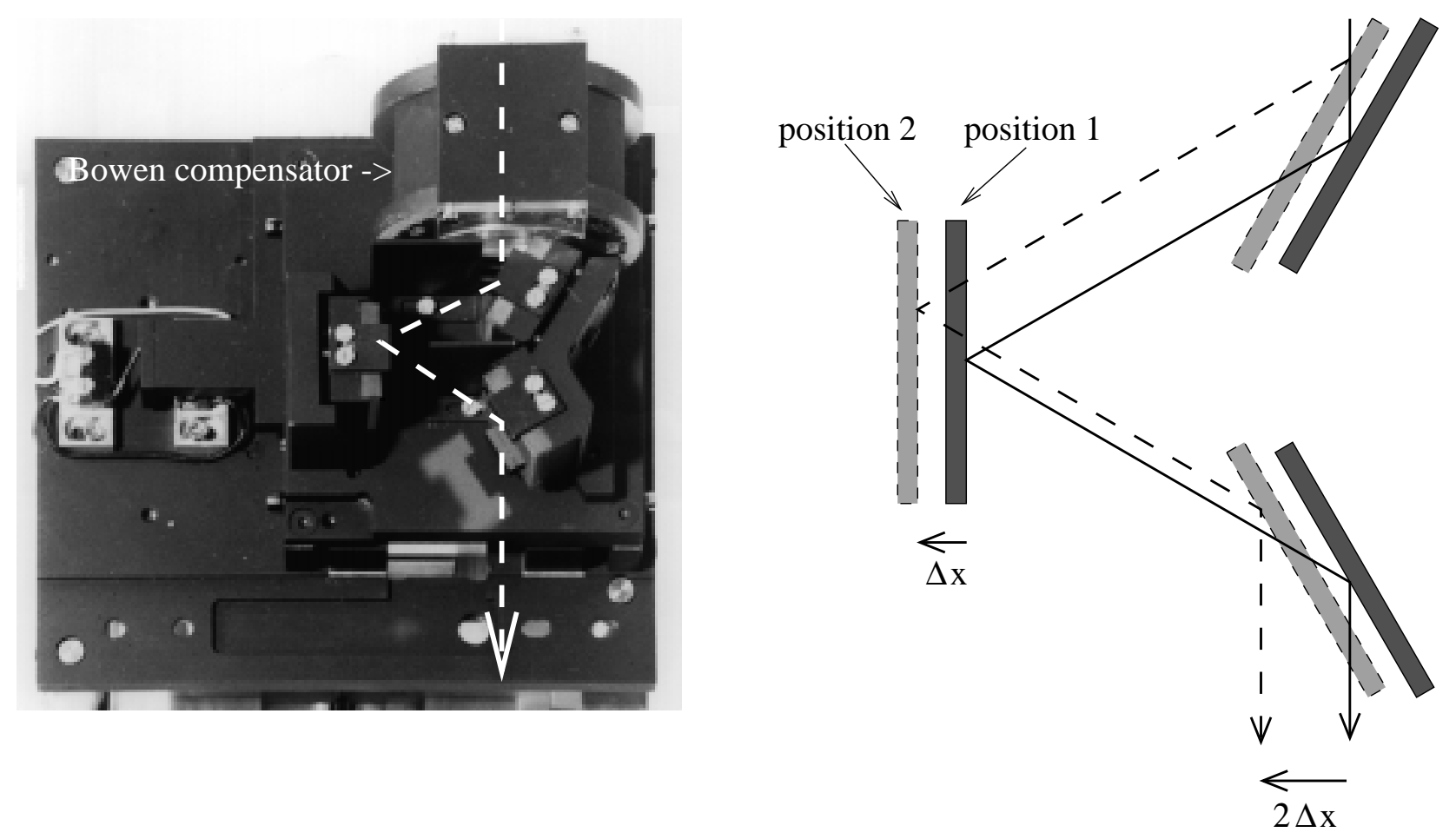

Fig. 2. Left: image scanner seen from above. The white dashed line marks the light path through the scanning device. The Bowen compensator is indicated. Right: scheme of the light paths through the scanner for two positions

\section{Instrumentation}

Spatial scanning takes full advantage of the GCT's spectrograph: high spatial and spectral resolution, wide observable wavelength range (near infrared to ultraviolet), simultaneous observations of several spectral lines (if requested on one CCD chip). Spatial scanning can be performed in different ways. When changing the pointing of the telescope it is necessary to move a large mass very fast and very precisely. This would require major changes in the telescope mount and drives. The GCT (Kneer et al. 1987) has a coudé mount, therefore drift scanning requires an image derotating device to align the East-West direction of the image always perpendicularly to the entrance slit of the spectrograph. Additionally, it would be necessary to control the drift speed in order to adapt it to the exposure time. The optical arrangement of the GCT and its spectrograph is given by Stix (1991).

These problems are avoided by MISC. It is mounted in front of the spectrograph's entrance slit and allows one to move the image perpendicularly across the entrance slit. The optical system of MISC consists of two major parts. The first one consists of three mirrors, which are arranged similarly as the active surfaces of a Dove prism. Figure 2 illustrates this device and its optical path. Shifting this mirror arrangement by a distance $\Delta x$ shifts the image by $2 \Delta x$. A minor disadvantage of this setup is that the optical axes of the telescope and the spectrograph are shifted against each other. This moves the light bundle across the grating and changes slightly its illumination. This problem could be solved by an accurate mechanical and optical adjustment and considering this effect in the data reduction.

The second part is a Bowen compensator (Koschinsky \& Kneer 1996 and references in there). This Bowen compensator consists of two $\lambda / 8$ retarder plates, which can be rotated against each other. Thus it is possible to produce phase retardations between $-\lambda / 4$ and $+\lambda / 4$. This device is used to compensate the phase changes from reflections at the mirrors thus preserving the excellent polarimetric properties of the GCT.

The mirrors and the Bowen compensator are mounted on a carriage running on a slippage-free recirculating ball spindle, which is driven by a stepping motor. One step of the motor moves the carriage by $2.03 \mu \mathrm{m}$, this yields $4.06 \mu \mathrm{m}$ image shift, corresponding to $00^{\prime \prime} 03$ in the telescopes focal plane. Figure 3 shows the complete MicroImage-Scanner on its mounting.

For polarimetry, a Stokes $-V$ polarimeter can be added to the setup. This polarimeter consists of an achromatic $(500-900 \mathrm{~nm}) \lambda / 4$ plate and a Savart plate (two crossed calcite rods). Beam splitting and beam diameter are optimized for the use with MISC and the CCD system described below. Even though the retarder plate is achromatic it is necessary to adjust it properly for the desired wavelength. It is known that achromatic retarder plates are not perfect over their wavelength range. 


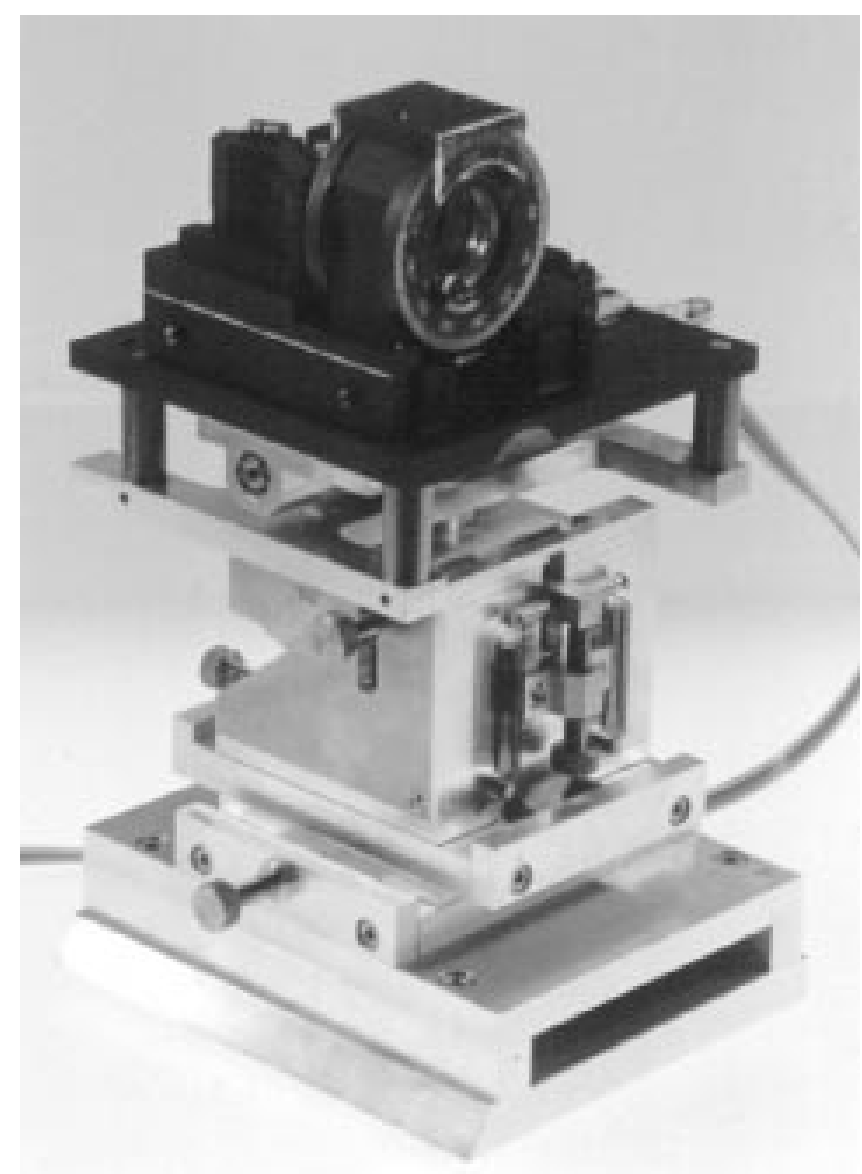

Fig. 3. Full view of the image scanner. The darker upper part is the scanning device, the lower part is the mounting which allows easy mechanical adjustment

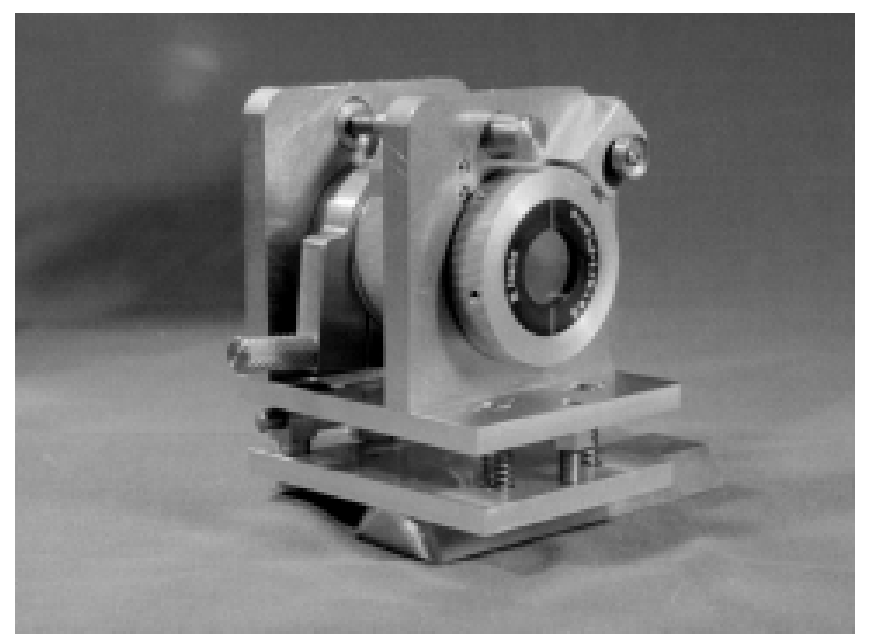

Fig. 4. The MISC Stokes $-V$ polarimeter

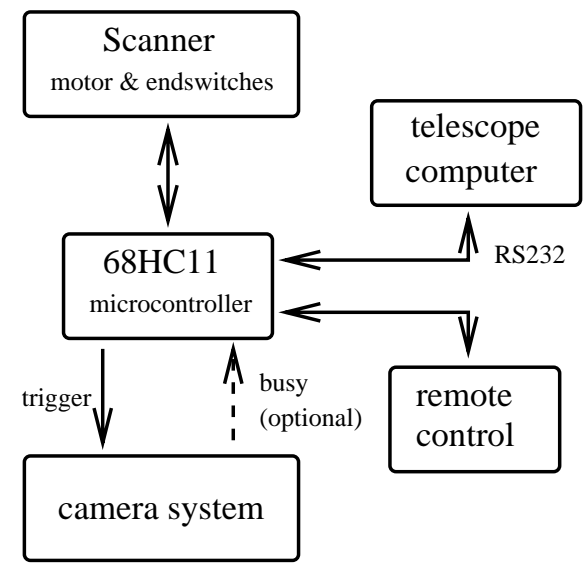

Fig. 5. Schematic diagram of the scanner control for its general mode

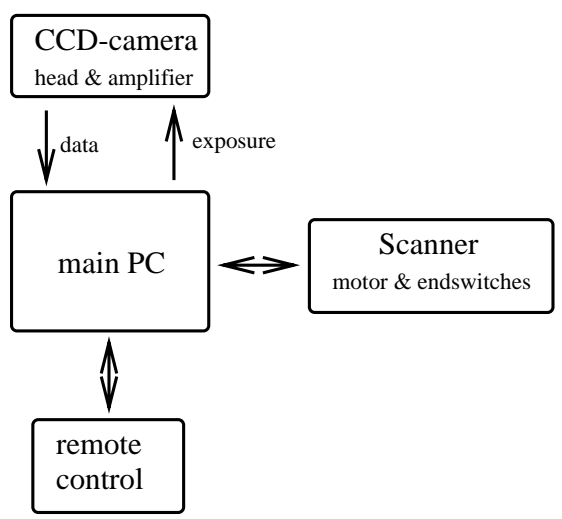

Fig. 6. Schematic diagram of the scanner control in its fast mode

The retardation angle and the orientation of the fast axis are slightly varying with wavelength. A reproducible adjustment helps to optimize the calibration. It is also possible to remove the $\lambda / 4$ plate from the polarimeter and move it back in place with reproducible accuracy and calibration. This allows to use retarder plates for various wavelengths or a $\lambda / 2$ plate to measure the other components of the Stokes vector.

The polarimeter is placed behind the spectrograph's entrance slit. In its present configuration as a Stokes- $V$ polarimeter it is possible to achieve a crosstalk as low as $0.5 \%$ at $770 \mathrm{~nm}$ and $3.5 \%$ at $617 \mathrm{~nm}$. In combination with the scanner the crosstalk varies between $1 \%$ and $6 \%$. The efficiency of the $\lambda / 4$ plate is better than $98 \%$. The MISCPolarimeter is shown in Fig. 4.

Fast scanning requires a camera system which is able to read out the CCD chip and to digitize the pixel values at high speed, and high dynamics and reliable accuracy. The FlamestarII system from LaVision satisfies these requirements. Originally it was designed for laser spectroscopy, but it fits very well into our system. It uses a Thomson 

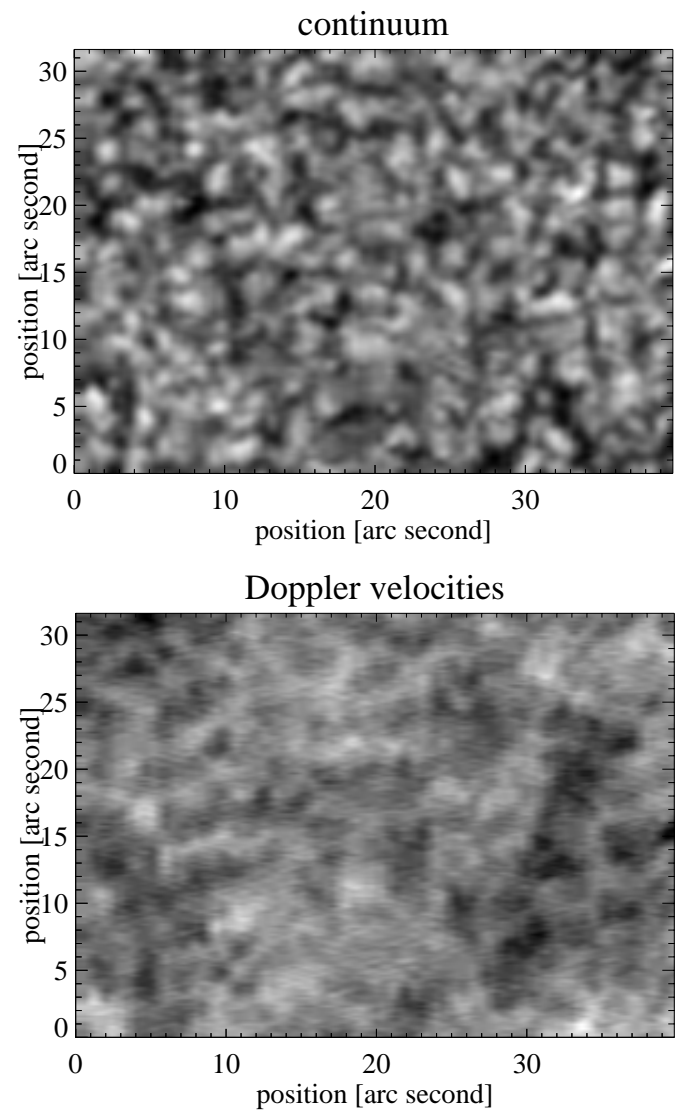

Fig. 7. Four examples of physical data calculated from one scan

TH 7863 FT CCD chip with $384 \times 286$ pixels. The pixel size of $23 \times 23 \mu \mathrm{m}^{2}$ fits the spatial and spectral resolution of the spectrograph. The chip is read out and digitized with $1.8 \mathrm{Mpixel} / \mathrm{s}$ at 12 bits dynamics. The chip is Peltier cooled. Due to the high readout speed the effective dynamic range of the camera is between 10 and 11 bits. The main control of the camera is based on a 486DX2/66 PC. LaVision provides a powerfull software for controlling and macro-programming of this system.

\section{Control}

The universal control of MISC and all attached systems, e.g. CCD cameras, has been complemented by a specialized and very efficient setup in which the $\mathrm{PC}$ of the $\mathrm{CCD}$ controls the whole system.

Figure 5 shows the general setup for using MISC with any camera that fits. The scanner is controlled by a $68 \mathrm{HC} 11$ microcontroller which also triggers the camera. The parameters for the scan, stepwidth, number of positions, time between two exposures etc., are transmitted by the telescopes main computer to the microcontroller, which then performs the scan. A remote control allows to move the scanner manually and to set the starting position.
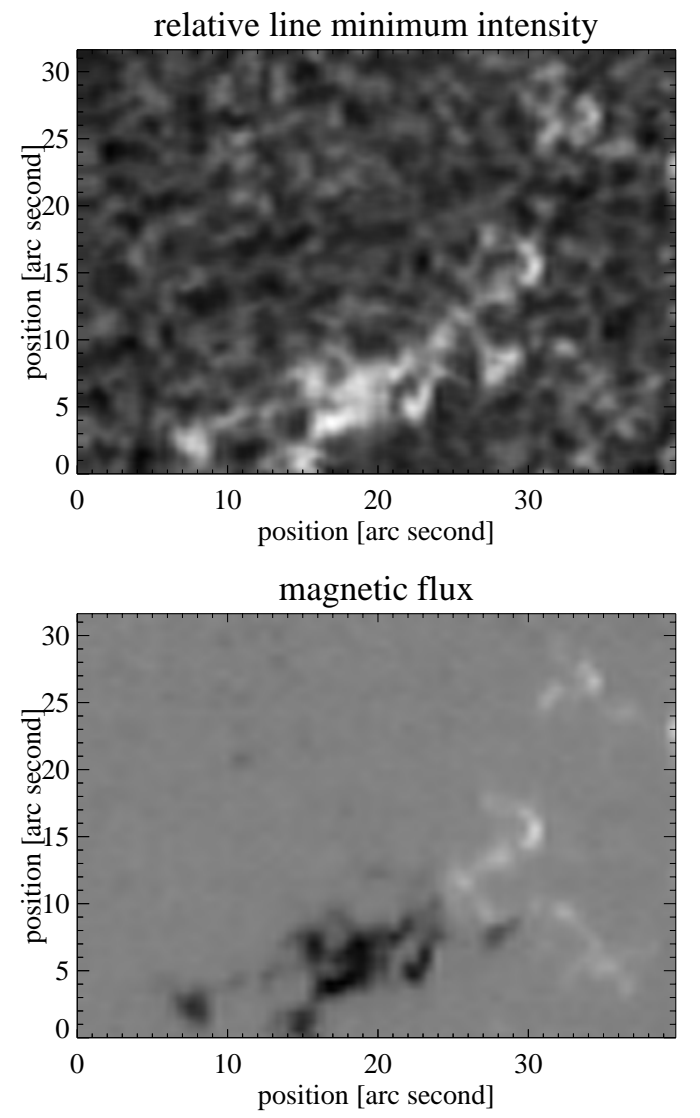

When MISC is used with the LaVision CCD it is easier and faster to use the scheme depicted in Fig. 6. The SC/CL3.1 software from LaVision can control stepping motors, therefore the scanner control can be integrated into the sequence of exposing and storing the spectra. The main PC serves primarily as the controller for the CCD, but integrates all functions of the MISC system. A simple remote control allows to operate MISC manually for certain purposes. To keep the scanning as fast as possible the camera PC is equipped with $64 \mathrm{MB}$ of RAM. The spectra obtained during the scan are stored in RAM and are transferred to a hard disc afterwards. The hard disc capacity of approximately 2 GB permits storage of more than 100 scans, depending on the number of spectra per scan and the use of binning.

\section{Performance}

To illustrate the perfomance of the system we describe some data taken from a typical oberving run. The used spectral line is FeI $630.25 \mathrm{~nm}$. Using MISC with the Stokes $-V$ polarimeter, the LaVision CCD, and the spectrographs slit width of 0.77 requires an exposure time of $200 \mathrm{~ms}$ at disk center. This exposure time provides $\approx 1000$ counts per pixel in the continuum. The noise $\sigma$ is $0.22 \%$, 
the achieved instrumental crosstalk is reduced to $2.6 \%$. The length of the slit is $\approx 32^{\prime \prime}$. Scanning a distance of $40^{\prime \prime}$ with 60 positions takes $24 \mathrm{~s}$ and storing the data onto the hard disc takes $\approx 15 \mathrm{~s}$. Hence, one scan takes $39 \mathrm{~s}$ at all and provides $13 \mathrm{MB}$ of spectral data. This allows to store 151 scans like this onto the disc. Taking the need for dark, flatfield an correlation scans into account, it is possible to store $\approx 120$ observing scans. In this case, scanning as fast as possible provides an observing time of $78 \mathrm{~min}$ for a single time series. Longer time intervals between two scans, binning, less positions etc. permit longer observing runs.

Figure 7 shows some images derived from the dataset of one scan taken near disk center. The upper left image is derived from the continuum near the observed spectral line. At the upper right is an image showing the minimum of the intensity in the spectral line relative to the local continuum. The Doppler image at the lower left was calculated from the wavelength of the spectral line's minimum. The Doppler velocities range from $-1400 \mathrm{~m} \mathrm{~s}^{-1}$ to $+1400 \mathrm{~m} \mathrm{~s}^{-1}$. The mean value is set to $0 \mathrm{~ms}^{-1}$. An image of the magnetic flux is shown at the lower right of Fig. 7. Assuming an effective resolution element of $0.7 \times 00^{\prime \prime} .7$ the magnetic flux ranges from $-2.410^{10} \mathrm{~Wb}$ to $1.610^{10} \mathrm{~Wb}$. The magnetic flux density within the resolution element ranges from $-119 \mathrm{mT}$ to $81 \mathrm{mT}^{1,2}$.

Similar maps, obtained with a correlation tracking device, can be found in Collados et al. (1996).

\section{Conclusion}

We presented a new, sophisticated instrument for multidimensional spectroscopy, consisting of the scanning device MISC, a Stokes- $V$ polarimeter and a fast CCD camera. Since its first preliminary installation at the GCT in July 1995, it has been in regular use and provides a wealth of data. The handling of the data and the first results will be discussed in a forthcoming publication.
Acknowledgements. We thank the mechanical and electronic workshops of the Universitäts-Sternwarte Göttingen where most of the hardware was designed and constructed. Advice from "La Vision 2D-Meßtechnik" is gratefully acknowledged.

The Gregory Coudé Telescope is operated by the Universitäts-Sternwarte Göttingen at the Spanish Observatorio del Teide of the Instituto de Astrofísica de Canarias. This work was supported by the Deutsche Forschungsgemeinschaft, through grant Kn 152/16-1,-2.

\section{References}

Ballesteros E., Collados M., Bonet J.A., Lorenzo F., Viera T., Reyes M., Rodríguez Hidalgo I., 1996, A\&AS 115, 353

Bendlin C., Volkmer R., Kneer F., 1992, A\&A 257, 817

Bonaccini D., Cavallini F., Righini A., 1989, A\&A 217, 368

Collados M., Rodríguez Hidalgo I., Ballesteros E., Ruiz Cobo B., Sánchez Almeida J., del Toro Iniesta J.C., 1996, A\&AS 115,367

Johannesson A., Bida T., Lites B., Scharmer G.B., 1992, A\&A 258,572

Kneer F., Schmidt W., Wiehr E., Wittmann A.D., 1987, Mitt. Astron. Ges. 68, 181

Koschinsky M., Kneer F., 1996, A\&AS 119, 171

Mein P., 1977, Solar Phys. 54, 45

Mein P., 1991, A\&A 248, 669

Stix M., 1991, The Sun. Springer Berlin, p. 77

Title A., 1984, Adv. Space Res. 4, No. 8, 67

Title A., Tarbell T., Simon G. and the SOUP Team, 1986, Adv. Space Res. 6, No. 8, 253

\footnotetext{
$11 \mathrm{~Wb}=1 \mathrm{Vs}=10^{8} \mathrm{Mx}$.

$21 \mathrm{~T}=1 \mathrm{~V} \mathrm{~s} \mathrm{~m}^{-2}=10^{4}$ Gauss.
} 